\title{
Turismo Comunitario Alternativa Para Lograr Emprendimientos Participativos Estudio De Caso: Determinación Comercial De Un Producto Turístico En El Cantón Suscal, Provincia Del Cañar Provincia De Cañar - Ecuador
}

\author{
Catalina Margarita Verdugo Bernal \\ Otto Fernando Balseca Sampedro \\ Carlos Renato Chávez Velásquez \\ Juan Carlos Carrasco Baquero \\ Juan Hugo Rodríguez Guerra
}

Docente - Investigador de la Facultad de Recursos Naturales

Escuela Superior Politécnica De Chimborazo - Ecuador

Rolando Fabián Zabala Vizuete

Docente - Investigador. Universidad Nacional De Chimborazo - Ecuador

\section{Verónica Lema Lema}

Estudiante, Escuela de Ingeniería en Ecoturismo, Facultad de Recursos

Naturales. Escuela Superior Politécnica De Chimborazo - Ecuador.

doi: 10.19044/esj.2016.v12n23p112 URL:http://dx.doi.org/10.19044/esj.2016.v12n23p112

\begin{abstract}
This research involved during a first phase a market study of the implementation of a tourist package in the Suscal County, province of Cañar, Ecuador, followed by a second phase, a Comprehensive Feasibility Study of the package. Using bibliographic research and a field tour, we described the package's commercial potential, technical, environmental, social, administrative, legal, economic, financial aspects. The study of tourism potential in the area identified the richness of an intangible cultural heritage, the existence of an ancestral collective memory alive in every community, and natural attractions of level I and II. The market study identified acceptance of the tourism package by $84 \%$ of domestic tourists and interest among $67 \%$ of foreign tourists, whose service whose preferences are: accommodation, food, cultural experience, transportation and information. Depending on the tourist profile, we will define and plan the technical design of tourism infrastructure and will conduct feasibility studies on legal, environmental and economic-financial aspects that will appear in a second
\end{abstract}


phase of the project. Despite its great potential, tourism in the province of Cañar has not achieved an optimal level, as reflected in the minimal fraction of GDP in cultural tourism, which in 2011 was 0.26\% (MCPEC, 2011:22), much lower than the national level. In Suscal County, this activity currently generates no income, but interest is recently being generated, while this study illustrates the potential of cultural tourism.

Keywords: Tourism, Ecuador

\section{Resumen}

La presente investigación propone: realizar en una primera etapa, el Estudio de Mercado para la implementación de un producto turístico en el cantón Suscal, provincia del Cañar, lo que establece la sensibilidad del mercado al servicio producido y la aceptación de consumo que tendría por lo que se estructuró el perfil del turista nacional y extranjero según sus preferencias; lo que aportará en una segunda fase al Estudio de Factibilidad integral del producto, es decir viabilidad técnica, ambiental, administrativalegal y económico-financiera que mediante la utilización de técnicas de investigación bibliográficas y de campo, permitieron evidenciar el potencial turístico del cantón y determinar la rentabilidad y beneficios del nuevo producto turístico. El estudio de potencialidad turística de la zona, permitió conocer la riqueza del patrimonio cultural inmaterial, evidenciando la existencia de una memoria colectiva ancestral viva en cada comunidad, además se identificaron atractivos naturales de jerarquía I, II. El estudio de mercado definió el nivel de aceptación en un $84 \%$ de turistas nacionales y el $67 \%$ de turistas extranjeros interesados por el producto turístico, cuyas preferencias de servicios son: alojamiento, alimentación, vivencia cultural, transporte e información. En función del perfil del turista se definirá y planificarán el diseño técnico de la infraestructura turística, y los estudios de factibilidad, legal, ambiental y económico - financiero que aparecerá en una segunda fase del proyecto. A pesar de su gran potencial en la provincia del Cañar el turismo no ha llegado al desarrollo óptimo, como se refleja en el PIB del año 2011 que fue de 0.26\% (MCPEC, 2011:22) solo del turismo cultural con respecto a nivel nacional, lo que indica que su progreso es mínimo, en el cantón Suscal el ejercicio de esta actividad no genera ningún ingreso económico pues recientemente se está dando a conocer y mediante el presente estudio se pretende evidenciar su potencialidad.

Palabras claves: Inventario turístico, estudio de mercado, oferta, demanda, producto turístico, turismo comunitario, Suscal 


\section{Introducción}

La (OMT, 2014:1), indica que el crecimiento y desarrollo de la actividad turística durante las últimas seis décadas ha experimentado una continua expansión y diversificación, convirtiéndose en uno de los sectores económicos de mayor envergadura y crecimiento del mundo, haciendo del mismo un factor clave para el progreso socioeconómico y una de las principales fuentes de ingresos de numerosos países en desarrollo. En el año 2014, las llegadas de turistas internacionales en el mundo crecieron un 4,3\%, además se previó un crecimiento entre 3\% y 4\% para el 2015, se estima para los siguientes años que la llegada de turistas a los países de economías emergentes, crezcan en un doble porcentaje de acuerdo a los países con economías avanzadas, esto también debido al impulso reciente de los países como Argentina, Ecuador y Colombia en América del Sur (OMT, 2015:2,7). Consecuentemente en el territorio ecuatoriano, las políticas públicas apuntan al impulso y cambio de la matriz productiva, abarcando los servicios del sector turístico que se ofertan en el mercado y la mejora continua de su calidad (SENPLADES, 2015: 49).

Se debe entender como matriz productiva, al conjunto que incluye los productos, los procesos productivos y las relaciones sociales resultantes de esos procesos, como en el sector turístico se tiene los tres componentes claves para este accionar, se convierte en un eje importante que permite a todos los involucrados en estas actividades formar parte del cambio (SENPLADES, 2012: 11,12). Teniendo en cuenta, la demanda o el mercado objetivo con su constante interacción y el producto que se oferta, se constituye así el sistema de turismo que abarca varios ejes y cadenas de valor, que al país le convienen explotar para obtener mejores réditos económicos, sin dejar de lado los ámbitos ambiental y cultural que se entrelazan para formar un producto integrado (Hernández, 2007:p.7).

En el Ecuador el turismo avanza paulatinamente, por ello la necesidad de crear productos de competitividad, con valor agregado y de calidad. Este sector toma cada vez más fuerza como un eje fundamental para el desarrollo socioeconómico del país y lo ha convertido en otro componente que genera grandes divisas económicas, nuevos fuentes de empleo y oportunidades de desarrollo socio cultural, constituyéndose así en una de las actividades aportantes al progreso (MINTUR, 2014:1). Se tienen grandes recursos naturales y culturales en el territorio, que con la adecuada gestión se logran posicionar turísticamente a nivel nacional e internacional, convirtiéndose en sitios de un alto rendimiento económico, solamente en base a las divisas que ingresan por concepto de la actividad turística sin embargo, recae la importancia de articular los servicios ofertados con los diferentes sectores productivos, para que los pequeños empresarios puedan tener su espacio dentro de este mercado (Calderón, 2015:7). Con los debidos 
procesos de planificación se puede plantear los objetivos que se deseen lograr en una determinada área, en este caso lograr el desarrollo local con la ayuda de la actividad turística, logrando así mediante la oportuna participación, planificación y gestión de los actores involucrados, un óptimo desarrollo de los proyectos planteados (Armijo, 2009:8).

La provincia del Cañar conocida por su riqueza arqueológica, natural y cultural, ha propiciado que el turismo vaya tomado espacio en la dinamización de la economía y contribuyendo a la promoción de este patrimonio, por lo cual se ha considerado como una alternativa viable para el desarrollo socioeconómico y el fomento de la participación social sin perder la identidad y la visión de una economía justa y solidaria (Mideros, 2015:4). Considerando de igual manera los derechos de las comunidades, pueblos y nacionalidades conforme con lo que establece la Constitución de la República del Ecuador en su artículo 57, en mantener proteger y desarrollar los conocimientos colectivos, a la vez que sean los mismos portadores del saber y veedores de los sitios y lugares naturales, culturales y ancestrales, quienes formen grupos estratégicos para hacer uso de estos recursos en productividad para alcanzar el Buen Vivir (Asamblea Nacional del Ecuador, 2008:27).

En este sentido, el producto turístico no es exclusivamente un lugar, sino también un conjunto de relaciones sectoriales y espaciales en las cuales se insertan realidades geográficas de muy diverso carácter, y que integran tanto espacios profundamente urbanizados junto con los espacios simbólicos y de relación social y cultural de los integrantes de ese espacio (Barrado, 2002:3,7). Es donde se empieza a desarrollar los usos sociales del patrimonio, entendiéndose como patrimonio los identificados tanto en el ámbito natural como cultural de un determinado espacio, que a su vez son vulnerables a los impactos turísticos que se generan por esta actividad. Recae aquí la importancia de realizar los estudios y debidos procesos de planificación para la creación de un producto turístico, que cuente con la participación de los mismos habitantes, veedores y gestores de este patrimonio (García, 1999: p.18, 19).

El 80\% de población del Suscal son indígenas (PDyOT provincia de Cañar, 2015:352), por lo que se destaca el patrimonio cultural a través de sus tradiciones, costumbres, vestimenta, gastronomía, religiosidad y otros que son de gran valor para la población local (Dores, 2011: p. 4). El patrimonio cultural esta entrelazado con la cotidianidad de la población, por ende se debe tener un alto sentido de respeto y apropiación para potencializarlo en uso de la actividad turística, lograr posicionar los diferentes recursos y revalorizar las prácticas culturales, fortalecerá la identidad de los habitantes, teniendo en cuenta la importancia de mantener viva en la memoria colectiva sus conocimientos ancestrales (Mullo, 2007: p.7). En cuanto al patrimonio 
natural existe un número menor de atractivos pero de igual relevancia, es primordial para la salud integral de los pobladores y de los posibles visitantes la conservación de estos sitios naturales, para lograr así una mejor experiencia de la convivencia turística cultural y natural.

Es así que la actual administración pública conjuntamente con la Mancomunidad del Pueblo Cañari se ha planteado el reto de convertir al cantón en un destino turístico competitivo con la implementación de productos turísticos que involucre la participación colectiva de las autoridades y los habitantes de las distintas comunidades del sitio (GADIPCS, 2013:34).

Lógicamente, la propuesta tiene una base técnica de determinación comercial, siendo indispensable analizar la demanda presente y la futura, a nivel nacional e internacional y las variables relevantes para su estimación. Las preferencias de la demanda permiten establecer en gran medida el tipo de producto turístico a diseñar y también lo hace el accionar de la competencia. Por ello se determina en la primera fase el perfil de la demanda, dejando para la segunda elementos como el marketing mix del producto y la factibilidad integral detallada en el resumen.

\section{Metodología}

En el presente estudio se aplicó las técnicas de investigación bibliográfica y de campo, a un nivel exploratorio, descriptivo, analítico y prospectivo. Para el cumplimiento de cada uno de los objetivos planteados se empleó la siguiente metodología:

\section{- Para la determinación del potencial turístico del cantón}

Para su cumplimiento se efectuó lo siguiente:

\section{Para la elaboración del inventario de atractivos turísticos se utilizó: Inventario de atractivos naturales}

Se realizó en base a la metodología del MINTUR (2004). Lo que consta de: a) Inventario y georeferenciación, b) Categorización, c) Valoración y, d) Evaluación y jerarquización. Las fichas aplicadas en recorridos de campo fueron validadas por un experto del MINTUR, el equipo de investigación y contrastadas en talleres participativos con los habitantes del territorio en estudio.

\section{Inventario de atractivos culturales}

Para el inventario del patrimonio cultural inmaterial se tomó en cuenta los ámbitos y subámbitos propuestos por el INPC (2011) para la clasificación del patrimonio cultural, ratificados en el "Instructivo para Fichas de Registro e Inventario del Patrimonio Cultural Inmaterial”. Consta de: a) Tradiciones y expresiones orales, b) Artes del espectáculo, c) Usos 
sociales, rituales y actos festivos, d) Conocimientos y usos relacionados con la naturaleza y el universo, e) Técnicas artesanales tradicionales. Las fichas aplicadas en el trabajo de campo fueron validadas por un experto del INPC, el equipo de investigación y contrastadas por la población.

- Para elaborar el estudio de Mercado.

Para el cumplimiento de este objetivo se revisaron fuentes de información primaria y secundaria como datos estadísticos e información adicional que proporcionó el GAD cantonal y la Mancomunidad. Adicionalmente se aplicaron en campo encuestas con un cuestionario de preguntas cerradas estructuradas por los técnicos en consulta con expertos y representantes comunitarios, las mismas que fueron tabuladas y sistematizadas el programa informático SPSS. Ver.3 y presentadas a través de gráficos estadísticos.

Análisis de la oferta analizó 1) Atractivos turísticos, 2) Planta turística, 3) Producto turístico.

Análisis de la demanda, determinó el perfil del turista:

Universo: Turistas nacionales y extranjeros que llegaron al Complejo Arqueológico de Ingapirca en el año 2014.

Muestra: Para su cálculo con la fórmula de la muestra para poblaciones finitas:

\section{Donde:}

$$
n=\frac{N(P * Q)}{(N-1)\left(\frac{e}{z}\right)^{2}+(P * Q)}
$$

$$
\begin{aligned}
& \mathbf{N}=\text { Universo de estudio } \\
& \mathbf{n}=\text { Tamaño de la muestra } \\
& \mathbf{p}=\text { Probabilidad de éxito }=0.5 \\
& \mathbf{q}=\text { Probabilidad de fracaso }=0.5 \\
& \mathbf{e}=\text { Margen de error }=0.035 \\
& \mathbf{z}=\text { Nivel de confianza }=1.96
\end{aligned}
$$

\section{Técnicas e instrumento}

Como instrumento se elaboró un cuestionario para turistas nacionales y extranjeros. La técnica utilizada para la investigación de campo fue la encuesta con preguntas cerradas y de opción múltiple en los idiomas español e inglés lo que permitió caracterizar la demanda definiendo el perfil del turista de cada segmento de acuerdo a sus respuestas.

\section{Confrontación oferta - demanda}

Para las proyecciones se utilizó el método de Incremento Compuesto descrito a continuación: 
$\operatorname{Cn}=\operatorname{Co}(1+\mathbf{i})^{\mathrm{n}}$

$\mathbf{C n}=$ incremento compuesto

$\mathbf{C o}=$ número de turistas potenciales

$\mathbf{i}=\quad$ índice de crecimiento turístico o poblacional

$\mathbf{n}=$ años a proyectar

Proyección de la demanda: Para la proyección de la demanda se fusionó los valores de los turistas nacionales y extranjeros, tomando como base el porcentaje de aceptación del proyecto en las encuestas.

Proyección de la oferta: Se proyectó para los cinco años posteriores al proyecto que permitirán determinar el abastecimiento del mercado, se utilizó el método del incremento compuesto.

Demanda potencial insatisfecha: Se aplicó la siguiente formula: $D P I=D-O$

Donde:

DPI = demanda potencial insatisfecha

$\mathbf{D}=$ demanda

$\mathbf{O}=$ oferta

Determinación de la cuota objetivo: Permitió realizar una aproximación para delimitar el tamaño del proyecto, en esta fase se proyectó los servicios de preferencia y actividades.

\section{Resultados}

- Inventariación de Recursos Naturales y Culturales

Cuadro 1: Matriz resumen de los atractivos turísticos naturales y culturales tangibles

\begin{tabular}{|c|c|c|c|c|c|c|}
\hline N. & $\begin{array}{c}\text { Nombre del } \\
\text { Atractivo }\end{array}$ & Categoría & Tipo & Subtipo & Jerarquía & Altitud \\
\hline 1 & $\begin{array}{c}\text { Laguna de } \\
\text { Chuyacocha }\end{array}$ & Sitios Naturales & $\begin{array}{c}\text { Ambientes } \\
\text { Lacustres }\end{array}$ & Lagunas & II & 2815 \\
\hline 2 & $\begin{array}{c}\text { Cascada de } \\
\text { Paila Wayku }\end{array}$ & Sitios Naturales & Ríos & Cascadas & I & 2556 \\
\hline 3 & $\begin{array}{c}\text { Bosque Nativo } \\
\text { Wayrapalte }\end{array}$ & Sitios Naturales & Bosques & $\begin{array}{c}\text { Bosque } \\
\text { Nublado } \\
\text { Occidental }\end{array}$ & II & 3251 \\
\hline 4 & $\begin{array}{c}\text { Cerro } \\
\text { Mesaloma }\end{array}$ & Sitios Naturales & Montaña & Cerro & II & 2980 \\
\hline 5 & Iglesia Matriz & $\begin{array}{c}\text { Manifestaciones } \\
\text { Culturales }\end{array}$ & Históricas & $\begin{array}{c}\text { Arquitectura } \\
\text { Religiosa }\end{array}$ & II & \\
\hline 6 & $\begin{array}{l}\text { Iglesia la } \\
\text { Dolorosa }\end{array}$ & $\begin{array}{c}\text { Manifestaciones } \\
\text { Culturales }\end{array}$ & Históricas & $\begin{array}{c}\text { Arquitectura } \\
\text { Religiosa }\end{array}$ & II & \\
\hline 7 & $\begin{array}{c}\text { Piedra } \\
\text { Calendario }\end{array}$ & $\begin{array}{c}\text { Manifestaciones } \\
\text { Culturales }\end{array}$ & Históricas & $\begin{array}{c}\text { Objetos } \\
\text { Arqueológicos }\end{array}$ & II & \\
\hline
\end{tabular}

Fuente: Investigación de campo, 2015

Realizado por: Verónica Lema. 
Cuadro 2: Matriz resumen de los atractivos culturales intangibles o patrimonio inmaterial.

\begin{tabular}{|c|c|c|c|c|c|}
\hline $\mathrm{N}^{\circ}$ & Código & Denominación & Ámbito & Subámbito & $\begin{array}{l}\text { Detalle del } \\
\text { subámbito }\end{array}$ \\
\hline 1 & $\begin{array}{l}\text { IM-03-07- } \\
\text { 50-002-15- } \\
00001\end{array}$ & Los Danzantes & $\begin{array}{l}\text { Artes del } \\
\text { Espectáculo }\end{array}$ & Danza & N/A \\
\hline 2 & $\begin{array}{c}\text { IM-03-07- } \\
50-002-15- \\
00002\end{array}$ & $\begin{array}{l}\text { Tucumán de } \\
\text { Cinta }\end{array}$ & $\begin{array}{l}\text { Artes del } \\
\text { Espectáculo }\end{array}$ & Danza & N/A \\
\hline 3 & $\begin{array}{c}\text { IM-03-07- } \\
50-002-15- \\
00003 \\
\end{array}$ & Taita Carnaval & $\begin{array}{c}\text { Artes del } \\
\text { Espectáculo }\end{array}$ & Música & N/A \\
\hline 4 & $\begin{array}{c}\text { IM-03-07- } \\
50-003-15- \\
00004\end{array}$ & Vestimenta & $\begin{array}{c}\text { Técnicas } \\
\text { artesanales } \\
\text { tradicionales }\end{array}$ & $\begin{array}{c}\text { Técnicas artesanales } \\
\text { tradicionales }\end{array}$ & $\begin{array}{l}\text { Indumentaria } \\
\text { cotidiana }\end{array}$ \\
\hline 5 & $\begin{array}{c}\text { IM-03-07- } \\
\text { 50-003-15- } \\
00005\end{array}$ & $\begin{array}{l}\text { Cantonización } \\
\text { de Suscal }\end{array}$ & $\begin{array}{c}\text { Usos sociales, } \\
\text { rituales y actos } \\
\text { festivos }\end{array}$ & Fiestas & Fiestas cívicas \\
\hline 6 & $\begin{array}{l}\text { IM-03-07- } \\
\text { 50-004-15- } \\
00006\end{array}$ & Chiviles & $\begin{array}{l}\text { Conocimientos y } \\
\text { usos } \\
\text { relacionados con } \\
\text { la naturaleza y el } \\
\text { universo }\end{array}$ & Gastronomía & $\begin{array}{c}\text { Gastronomía } \\
\text { cotidiana, festiva } \\
\text { y artesanal }\end{array}$ \\
\hline 7 & $\begin{array}{l}\text { IM-03-07- } \\
\text { 50-004-15- } \\
00007\end{array}$ & $\begin{array}{l}\text { Dulce de } \\
\text { zambo }\end{array}$ & $\begin{array}{l}\text { Conocimientos y } \\
\text { usos } \\
\text { relacionados con } \\
\text { la naturaleza y el } \\
\text { universo } \\
\end{array}$ & Gastronomía & $\begin{array}{l}\text { Gastronomía } \\
\text { cotidiana }\end{array}$ \\
\hline 8 & $\begin{array}{l}\text { IM-03-07- } \\
50-004-15- \\
00008\end{array}$ & Chumales & $\begin{array}{l}\text { Conocimientos y } \\
\text { usos } \\
\text { relacionados con } \\
\text { la naturaleza y el } \\
\text { universo } \\
\end{array}$ & Gastronomía & $\begin{array}{l}\text { Gastronomía } \\
\text { cotidiana }\end{array}$ \\
\hline 9 & $\begin{array}{l}\text { IM-03-07- } \\
50-004-15- \\
00009\end{array}$ & Navus kururu & $\begin{array}{c}\text { Conocimientos y } \\
\text { usos } \\
\text { relacionados con } \\
\text { la naturaleza y el } \\
\text { universo }\end{array}$ & Gastronomía & $\begin{array}{l}\text { Gastronomía } \\
\text { cotidiana }\end{array}$ \\
\hline 10 & $\begin{array}{l}\text { IM-03-07- } \\
50-004-15- \\
00010\end{array}$ & $\begin{array}{c}\text { Zarza de } \\
\text { zambo }\end{array}$ & $\begin{array}{l}\text { Conocimientos y } \\
\text { usos } \\
\text { relacionados con } \\
\text { la naturaleza y el } \\
\text { universo } \\
\end{array}$ & Gastronomía & $\begin{array}{l}\text { Gastronomía } \\
\text { cotidiana }\end{array}$ \\
\hline 11 & $\begin{array}{c}\text { IM-03-07- } \\
\text { 50-005-15- } \\
00011\end{array}$ & Caja & $\begin{array}{c}\text { Técnicas } \\
\text { artesanales } \\
\text { tradicionales }\end{array}$ & $\begin{array}{c}\text { Técnicas artesanales } \\
\text { tradicionales }\end{array}$ & $\begin{array}{l}\text { Instrumentos } \\
\text { musicales de } \\
\text { percusión }\end{array}$ \\
\hline 12 & $\begin{array}{l}\text { IM-03-07- } \\
\text { 50-005-15- } \\
00012\end{array}$ & $\begin{array}{l}\text { Artesanía } \\
\text { suscaleña }\end{array}$ & $\begin{array}{c}\text { Técnicas } \\
\text { artesanales } \\
\text { tradicionales }\end{array}$ & $\begin{array}{c}\text { Técnicas artesanales } \\
\text { tradicionales }\end{array}$ & Textilería \\
\hline
\end{tabular}

Fuente: Investigación de campo, 2015.

Realizado por: Verónica Lema. 


\section{- $\quad$ Estudio de Mercado}

\section{Análisis de la oferta turística}

\section{Planta turística de la Competencia}

El territorio presenta una variedad importante de establecimientos que atienden a la demanda turística directa o indirectamente y a la vez ofrece atractivos turísticos naturales y culturales (Cuadro 1 y 2) y que los suscaleños promocionan a locales y foráneos.

La información en cuanto a los prestadores de servicios turísticos se obtuvo del catastro turístico brindado por el departamento de Cultura, Social y Turismo del Gobierno Autónomo Descentralizado Intercultural Participativo Cultural de Suscal, debido a que la planta turística dentro de la zona de estudio es muy escasa, se recurrió a la Unidad de Turismo del cantón Cañar y el cantón El Tambo con la finalidad de obtener otras alternativas para los turistas en los poblados más cercanos al cantón. A continuación se detallan:

Cuadro 3: Matriz resumen, establecimientos de la planta turística de los cantones Suscal,

Cañar y El Tambo.

\begin{tabular}{|c|c|c|c|}
\hline CANTÓN & ESTABLECIMIENTO & CATEGORÍA & NÚMERO \\
\hline \multirow{2}{*}{ Suscal } & RESTAURANTES & Tercera & 5 \\
\cline { 2 - 4 } & BAR - CAFETERIAS & Tercera & 2 \\
\hline \multirow{3}{*}{ Cañar } & ALOJAMIENTOS & Tercera & 5 \\
\cline { 2 - 4 } & \multirow{2}{*}{ RESTAURANTES } & Cuarta & 8 \\
\cline { 2 - 4 } & & Tercera & 15 \\
\hline \multirow{3}{*}{ El Tambo } & ALOJAMIENTO & Tercera & 4 \\
\cline { 2 - 4 } & \multirow{2}{*}{ RESTAURANTES } & Cuarta & 6 \\
\cline { 2 - 4 } & & Tercera & 6 \\
\hline
\end{tabular}

Fuente: Catastro turístico del Cantón Cañar, 2015.

Realizado por: Catalina Verdugo Bernal.

\section{b. Producto turístico}

En el área de estudio se ofertan las siguientes líneas de productos, que no han sido aprovechadas.

Cuadro 4.: Líneas de productos existentes en el área de estudio.

\begin{tabular}{|c|c|c|c|}
\hline $\begin{array}{c}\text { LÍNEAS DE } \\
\text { PRODUCTOS }\end{array}$ & $\begin{array}{l}\text { VARIEDAD } \\
\text { PRODUCTOS }\end{array}$ & $\begin{array}{c}\text { NOMBRE } \\
\text { PRODUCTO }\end{array}$ & ACTIVIDADES \\
\hline \multirow[b]{6}{*}{ Turismo cultural } & \multirow{5}{*}{ Gastronomía } & Chiviles & \multirow{5}{*}{$\begin{array}{l}\text { Productos de consumo en festividades } \\
\text { locales }\end{array}$} \\
\hline & & Humitas & \\
\hline & & Dulce de zambo & \\
\hline & & Navus kururu & \\
\hline & & Zarza de zambo & \\
\hline & Fiestas Populares & Taita Carnaval & $\begin{array}{c}\text { Recorrido cultural por comunidades } \\
\text { vestidos de carnavaleros y de traje típico } \\
\text { acompañados del principal instrumento } \\
\text { musical “caja o tambora”, pingullos y a } \\
\text { viva voz van cantando en coro. En las } \\
\text { comunidades se brinda una gran variedad } \\
\text { de platos típicos de la zona }\end{array}$ \\
\hline
\end{tabular}




\begin{tabular}{|c|c|c|c|}
\hline & & Cantonización & $\begin{array}{c}\text { Diferentes programas culturales y } \\
\text { deportivos se llevan a cabo en honor a la } \\
\text { cantonización siendo las principales: } \\
\text { elección y proclamación de la reina y } \\
\text { ñusta, feria gastronómica y agroecológica, } \\
\text { desfile cívico, entre otros }\end{array}$ \\
\hline & & San Jacinto & $\begin{array}{c}\text { De igual manera se llevan a cabo } \\
\text { diferentes programas religiosos, culturales } \\
\text { y deportivos, resaltando: la novena, } \\
\text { procesión y misa en honor al patrono } \\
\text { además de festival de comida típica y de } \\
\text { danzas folclóricas }\end{array}$ \\
\hline & & Dolorosa & $\begin{array}{l}\text { Celebración de misa en nombre de la } \\
\text { virgen, escarmuzas, Tucumán forma parte } \\
\text { de estas festividades. }\end{array}$ \\
\hline \multirow{4}{*}{$\begin{array}{l}\text { Ecoturismo y } \\
\text { turismo de } \\
\text { naturaleza }\end{array}$} & \multirow{2}{*}{$\begin{array}{c}\text { Reservas y } \\
\text { bosques privados }\end{array}$} & $\begin{array}{c}\text { Bosque Nativo } \\
\text { Wayrapalte }\end{array}$ & Treking, \\
\hline & & $\begin{array}{c}\text { Cerro } \\
\text { Mesaloma } \\
\end{array}$ & Treking, camping hacia el cerro. \\
\hline & \multirow{2}{*}{$\begin{array}{l}\text { Ríos, lagos, } \\
\text { lagunas y } \\
\text { cascadas }\end{array}$} & $\begin{array}{l}\text { Cascada Paila } \\
\text { Wayku }\end{array}$ & $\begin{array}{c}\text { Treking, camping hacia el bosque nativo, } \\
\text { observación de flora y fauna local } \\
\text { existente. }\end{array}$ \\
\hline & & $\begin{array}{c}\text { Laguna } \\
\text { Chuyacucha } \\
\end{array}$ & Treking, camping alrededor de la laguna. \\
\hline $\begin{array}{l}\text { Turismo de } \\
\text { salud }\end{array}$ & $\begin{array}{l}\text { Medicina } \\
\text { Ancestral }\end{array}$ & $\begin{array}{l}\text { Medicina } \\
\text { Ancestral: } \\
\text { curanderas }\end{array}$ & Limpias \\
\hline
\end{tabular}

Fuente: Investigación de campo, 2015.

Realizado por: Verónica Lema.

\section{Análisis de la demanda turística}

Segmentación del mercado

Universo de estudio: El Complejo Arqueológico Ingapirca en el año 2014 recibió un total 111329 turistas tanto nacionales como extranjeros. De los cuales 84182 fueron nacionales y 27147 fueron extranjeros.

Cálculo de la muestra: se utilizó la fórmula de la muestra para poblaciones finitas:

\section{Donde:}

$$
n=\frac{N(P * Q)}{(N-1)\left(\frac{e}{z}\right)^{2}+(P * Q)}
$$
$\mathbf{N}=\quad$ Universo de estudio
$\mathbf{n}=\quad$ Tamaño de la muestra
$\mathbf{p}=\quad$ Probabilidad de éxito $=0.5$
$\mathbf{q}=$ Probabilidad de fracaso $=0.5$ 
$\mathbf{e}=\quad$ Margen de error $=0.035$

$\mathbf{z}=\quad$ Nivel de confianza $=1.96$

La muestra se obtuvo del universo y luego mediante la estratificación se determinó el número de encuesta a aplicarse para cada segmento:

$n=111329 * 0.5 * 0.5$

$(111329-1)(0.035) 2+0.5 * 0.5$

1.96

$n=27832$.

2535751

$n=779$

Distribución de encuestas: nacionales 589 (75\%) y extranjeros 190 (25\%).

Se estableció 778 encuestas que se aplicaron en el Complejo Arqueológico Ingapirca; de la cual al efectuar la estratificación se obtuvo 589 encuestas para los turistas nacionales y 190 para extranjero. Se utilizó como instrumento una encuesta de opción múltiple aplicado a turistas de habla hispana y de habla inglesa.

\section{Turistas nacionales y extranjeros}

\section{Edad}

Tabla $\mathbf{N}^{\mathbf{0}}$ 1. Rango de edad de los turistas nacionales y extranjeros

\begin{tabular}{|c|c|c|c|c|c|}
\hline \multicolumn{2}{|c|}{ Turistas nacionales } & \multicolumn{2}{c|}{ Turistas extranjeros } \\
\hline Variables & $\mathrm{N}^{\circ}$ & $\%$ & Variables & $\mathrm{N}^{\circ}$ & $\%$ \\
\hline Menor de 18 años & 132 & 22,4 & Menor de 18 años & 8 & 4,2 \\
\hline Entre 18 - 38 años & 266 & 45,2 & Entre 18 - 38 años & 87 & 45,8 \\
\hline Entre 39- 59- años & 124 & 21,1 & Entre 39- 59- años & 71 & 37,4 \\
\hline Mayor de 60 años & 67 & 11,4 & Mayor de 60 años & 24 & 12,6 \\
\hline TOTAL & 589 & $100 \%$ & TOTAL & 190 & $100 \%$ \\
\hline
\end{tabular}

Fuente: Investigación de campo.

Realizado por: Verónica Lema.

Según la encuesta realizada el rango de edad que lidera en cuanto a los turistas nacionales es de 18 - 38 años con el $45 \%$, seguido por los menores de 18 años con el 23\%, de 39-59 años con el 21\%, mientras que los mayores de 60 años con el 11\%. Mientras que los turistas extranjeros se determinó que el rango de edad de entre 18-38 años con el más alto porcentaje es de $46 \%$, seguido por el de 39-59 años con el $37 \%$, los mayores de 60 años con un 13\% y los menores de 18 años con el 4\%. Esto indica que las actividades a realizarse deben estar orientadas a personas de estos rangos de edad. 
Género

Tabla $\mathbf{N}^{\mathbf{0}}$ 2. Género de los turistas nacionales.

\begin{tabular}{|c|c|c|c|c|c|}
\hline \multicolumn{3}{|c|}{ Turista nacional } & \multicolumn{3}{c|}{ Turista extranjero } \\
\hline Variables & $\mathrm{N}^{\circ}$ & $\%$ & Variables & $\mathrm{N}^{\circ}$ & $\%$ \\
\hline Masculino & 302 & 51,3 & Masculino & 97 & 51,1 \\
\hline Femenino & 287 & 48,7 & Femenino & 93 & 48,9 \\
\hline TOTAL & 589 & $100 \%$ & TOTAL & 190 & $100 \%$ \\
\hline
\end{tabular}

Fuente: Investigación de campo.

Realizado por: Verónica Lema.

Interpretación: Los resultados obtenidos a partir de la aplicación de la encuesta indican que el $51 \%$ de los turistas nacionales son de género masculino, mientras que el $49 \%$ es de género femenino. El porcentaje de variación con respecto al género del turista extranjero se determinó que el $51 \%$ son masculinos y el $49 \%$ femenino.

\section{Procedencia}

Tabla $\mathbf{N}^{\circ}$ 3. Procedencia de los turistas nacionales.

\begin{tabular}{|c|c|c|c|c|c|}
\hline \multicolumn{3}{|c|}{ Turista nacional } & \multicolumn{3}{|c|}{ Turista extranjero } \\
\hline Variables & $\mathrm{N}^{\circ}$ & $\%$ & Variables & $\mathrm{N}^{\circ}$ & $\%$ \\
\hline Costa & 201 & 34,1 & Suiza & 6 & 3,2 \\
\hline Sierra & 377 & 64,0 & EEUU & 52 & 27,4 \\
\hline Amazonía & 11 & 1,9 & España & 6 & 3,2 \\
\hline Insular & 0 & 0,0 & Alemania & 36 & 18,9 \\
\hline TOTAL & 589 & $100 \%$ & Francia & 35 & 18,4 \\
\hline & & & Dinamarca & 10 & 5,3 \\
\hline & & & Canadá & 12 & 6,3 \\
\hline & & & Inglaterra & 7 & 3,7 \\
\hline & & & Argentina & 6 & 3,2 \\
\hline & & & Netherland & 4 & 3,2 \\
\hline & & & Turquia & 3 & 2,1 \\
\hline & & & Colombia & 3 & 1,6 \\
\hline & & & Malasia & 3 & 1,6 \\
\hline & & & Corea & 4 & 2,1 \\
\hline & & & TOTAL & 190 & $100 \%$ \\
\hline
\end{tabular}

Fuente: Investigación de campo.

Realizado por: Verónica Lema.

En los resultados obtenidos de la encuesta se determinó que la procedencia de los turistas nacionales por regiones donde el 64\% provienen de la Sierra, el 34\% de la Costa, el 2\% es de la Amazonía, mientras que del Insular no se registra ningún visitante. Mediante la encuesta formulada para determinar la procedencia de los turistas extranjeros se evidencio que el $27 \%$ son de Estados Unidos, seguido por el 19\% que son de Alemania y Francia respectivamente, un 7\% de Canadá, otro 5\% de Dinamarca, un 4\% de Inglaterra, el 3\% de España, Argentina y Netherland individualmente, y 
finalmente el 2\% son de Turquía, Colombia, Corea y Malasia respectivamente.

\section{Nivel de educación}

Tabla $\mathbf{N}^{\mathbf{0}}$ 4. Nivel de educación de los turistas nacionales y extranjeros.

\begin{tabular}{|c|c|c|c|c|c|}
\hline \multicolumn{3}{|c|}{ Turista nacional } & \multicolumn{3}{c|}{ Turista extranjero } \\
\hline Variables & $\mathrm{N}^{\circ}$ & $\%$ & Variables & $\mathrm{N}^{\circ}$ & $\%$ \\
\hline Primaria & 160 & 27,2 & & 9 & 4,7 \\
\hline Secundaria & 210 & 35,7 & & 14 & 7,4 \\
\hline Superior & 192 & 32,6 & & 139 & 73,2 \\
\hline Posgrado & 27 & 4,6 & & 28 & 14,7 \\
\hline TOTAL & 589 & $100 \%$ & & 190 & $100 \%$ \\
\hline
\end{tabular}

Fuente: Investigación de campo.

Realizado por: Verónica Lema

A través de la encuesta realizada a los turistas nacionales se evidencia que el nivel de educación o instrucción de los visitantes es liderada por la formación secundaria con el 36\%, seguida por la superior con 33\%, la primaria es representado con el $27 \%$ y el postgrado con un $4 \%$. Los turistas extranjeros encuestados referente al nivel de educación con el porcentaje más alto es la superior con un $73 \%$, el $15 \%$ son de postgrado, el $7 \%$ de la secundaria y finalmente el $5 \%$ de la primaria.

\section{Motivo de viaje}

Tabla $N^{\circ}$ 5. Motivo de viaje de los turistas nacionales.

\begin{tabular}{|c|c|c|c|c|c|}
\hline \multicolumn{2}{|c|}{ Turismo nacional } & \multicolumn{3}{c|}{ Turista extranjero } \\
\hline Variables & $\mathrm{N}^{\circ}$ & $\%$ & Variables & $\mathrm{N}^{\circ}$ & $\%$ \\
\hline Estudio & 75 & 12,7 & Turismo & 187 & 98,4 \\
\hline Trabajo & 76 & 12,9 & Estudio & 1 & 0,5 \\
\hline Descanso & 73 & 12,4 & Negocio & 2 & 1,1 \\
\hline Distracción & 119 & 20,2 & & & \\
\hline Vacaciones & 246 & 41,8 & & & \\
\hline TOTAL & 589 & $100 \%$ & TOTAL & 190 & $100 \%$ \\
\hline
\end{tabular}

Fuente: Investigación de campo.

Realizado por: Verónica Lema

A partir de la encuesta aplicada se aprecia que cuando realizan viajes existen diferentes motivos en este caso el $42 \%$ de los turistas nacionales viajan por vacaciones, el $20 \%$ por distracción, el 13\% estudio y trabajo respectivamente, mientras que el $12 \%$ lo hacen por descanso. El motivo por el cual generalmente viajan los turistas extranjeros son el 98\% por turismo, el $1 \%$ por negocio y otro $1 \%$ por estudio. Lo que evidencia que este segmento de la demanda viaja motivados por nuevos destinos. 


\section{Con quién viaja}

Tabla $\mathbf{N}^{\circ} \mathbf{6}$. Viajes que realizan los turistas nacionales.

\begin{tabular}{|c|c|c|c|c|c|}
\hline \multicolumn{2}{|c|}{ Turista nacional } & \multicolumn{2}{c|}{ Turista extranjero } \\
\hline Variables & $\mathrm{N}^{\circ}$ & $\%$ & Variables & $\mathrm{N}^{\circ}$ & $\%$ \\
\hline Familia & 279 & 47,4 & Familia & 35 & 18,4 \\
\hline Amigos & 186 & 31,6 & Amigos & 83 & 43,7 \\
\hline Pareja & 47 & 8,0 & Pareja & 48 & 25,3 \\
\hline Solo & 11 & 1,9 & Solo & 23 & 12,1 \\
\hline Compañero de trabajo & 66 & 11,2 & Compañero de trabajo & 1 & 0,5 \\
\hline TOTAL & 589 & $100 \%$ & TOTAL & 190 & $100 \%$ \\
\hline
\end{tabular}

Fuente: Investigación de campo.

Realizado por: Verónica Lema.

El $47 \%$ de los posibles clientes indican que realizan sus viajes en familia, un $32 \%$ entre amigos, el $11 \%$ con compañeros de trabajo, otro $8 \%$ con la pareja, mientras que el $2 \%$ restante lo hace solo. Al momento de viajar los turistas extranjeros prefieren viajar en un $44 \%$ entre amigos, un $26 \%$ en pareja, el $18 \%$ en familia, el $12 \%$ solo y el $1 \%$ restante con compañero de trabajo.

\section{Frecuencia anual de vacaciones}

Tabla $\mathbf{N}^{\circ}$ 7. Frecuencia de viajes de los turistas nacionales.

\begin{tabular}{|c|c|c|c|c|c|}
\hline \multicolumn{3}{|c|}{ Turista nacional } & \multicolumn{3}{c|}{ Turista extranjero } \\
\hline Variables & $\mathrm{N}^{\circ}$ & $\%$ & Variables & $\mathrm{N}^{\mathrm{o}}$ & $\%$ \\
\hline 1 vez & 256 & 43,5 & 1 vez & 81 & 42,6 \\
\hline 2 veces & 221 & 37,5 & 2 veces & 83 & 43,7 \\
\hline 3 veces & 112 & 19,0 & 3 veces & 26 & 13,7 \\
\hline TOTAL & 589 & $100 \%$ & TOTAL & 190 & $100 \%$ \\
\hline
\end{tabular}

Fuente: Investigación de campo.

Realizado por: Verónica Lema.

En cuanto a la frecuencia de viajes se puede observar que el $43 \%$ del total de turistas encuestados viajan una vez al año, seguido por el $38 \%$ que lo suelen hacer dos veces y el 19\% restante tres veces. Los turistas extranjeros encuestados indican que por lo menos al año realizan en un $44 \%$ su viajes o salen de vacaciones dos veces, el $42 \%$ una vez y el $14 \%$ restante tres veces.

\section{Conoce Suscal}

Tabla $\mathbf{N}^{\mathbf{0}} \mathbf{8}$. Conocimiento acerca del cantón.

\begin{tabular}{|c|c|c|c|c|c|}
\hline \multicolumn{3}{|c|}{ Turista nacional } & \multicolumn{3}{c|}{ Turista extranjero } \\
\hline Variables & $\mathrm{N}^{\circ}$ & $\%$ & Variables & $\mathrm{N}^{\circ}$ & $\%$ \\
\hline SI & 132 & 22,4 & SI & 9 & 4,7 \\
\hline NO & 457 & 77,6 & NO & 181 & 95,3 \\
\hline TOTAL & 589 & $100 \%$ & TOTAL & 190 & $100 \%$ \\
\hline
\end{tabular}

Fuente: Investigación de campo.

Realizado por: Verónica Lema. 
Se determinó que el 78\% de los turistas nacionales no han visitado y tampoco han oído hablar acerca del cantón Suscal, el 22\% restante si lo han frecuentado. El 95\% de los turistas extranjeros encuestados mencionan que desconocen sobre la existencia de este cantón y tampoco lo han visitado, mientras que el 5\% indica que por lo menos ha pasado por este sector o han escuchado hablar de él.

\section{Predisposición por visitar Suscal}

Tabla No 9. Predisposición para visitar Suscal

\begin{tabular}{|c|c|c|c|c|c|}
\hline \multicolumn{3}{|c|}{ Turista nacional } & \multicolumn{3}{c|}{ Turista extranjero } \\
\hline Variables & $\mathrm{N}^{\circ}$ & $\%$ & Variables & $\mathrm{N}^{\circ}$ & $\%$ \\
\hline SI & 494 & 83,9 & SI & 128 & 67,4 \\
\hline NO & 95 & 16,1 & NO & 62 & 32,6 \\
\hline TOTAL & 589 & $100 \%$ & TOTAL & 190 & $100 \%$ \\
\hline
\end{tabular}

Fuente: Investigación de campo.

Realizado por: Verónica Lema

Los turistas nacionales en un 84\% desean visitar el cantón Suscal mientras que el $16 \%$ no le desea. En cuanto al turista extranjero, se determinó que al $67 \%$ de la población le gustaría visitarlo, mientras que el $33 \%$ no se dispone a visitar este cantón. Detalle a tomar en cuenta al momento de estructurar productos que operen en la zona.

\section{Actividades de preferencia}

Tabla $\mathbf{N}^{\mathbf{0}} \mathbf{1 0}$. Actividades a realizar los turistas nacionales

\begin{tabular}{|c|c|c|c|c|}
\hline & \multicolumn{2}{|c|}{ Turista nacional } & \multicolumn{2}{c|}{ Turista extranjero } \\
\hline Variables & $\mathrm{N}^{\circ}$ & $\%$ & $\mathrm{~N}^{\circ}$ & $\%$ \\
\hline Caminatas & 87 & 17,6 & 21 & 16,4 \\
\hline Cabalgatas & 41 & 8,3 & 7 & 5,5 \\
\hline Ciclismo & 46 & 9,3 & 4 & 3,1 \\
\hline Camping & 32 & 6,5 & 6 & 4,7 \\
\hline Observación de flora y fauna & 61 & 12,3 & 41 & 32,0 \\
\hline Participar en festividades locales & 53 & 10,7 & 8 & 6,3 \\
\hline Degustar de comida típica & 98 & 19,8 & 12 & 9,4 \\
\hline Turismo cultural & 76 & 15,4 & 29 & 22,7 \\
\hline TOTAL & 494 & $100 \%$ & 128 & $100 \%$ \\
\hline
\end{tabular}

Fuente: Investigación de campo.

Realizado por: Verónica Lema

El 20\% de los turistas nacionales encuestados han indicado que en caso de visitar el cantón Suscal preferirían degustar de la comida típica, el $18 \%$ quiere realizar actividades como la caminata, el 15\% desea participar en el turismo cultural, un 12\% quiere la observación de flora y fauna, otro $11 \%$ participar en festividades locales, un 9\% ciclismo, el 8\% cabalgatas y el 7\% restante camping. Los turistas extranjeros encuestados mencionan que en 
caso de visitar el cantón Suscal le gustaría realizar actividades en un 32\% en la observación de flora y fauna, el 23\% participar del turismo cultural, un $16 \%$ desea realizar caminatas, otro 9\% quiere degustar la comida típica del sitio, el 6\% gusta en participar en festividades locales y realizar cabalgatas respectivamente, un 5\% camping y un 3\% ciclismo.

\section{Duración de la visita}

Tabla $\mathbf{N}^{\mathbf{0}}$ 11. Tiempo de visita de turistas nacionales.

\begin{tabular}{|c|c|c|c|c|}
\hline & \multicolumn{2}{|c|}{ Turista nacional } & \multicolumn{2}{c|}{ Turista extranjero } \\
\hline Variables & $\mathrm{N}^{\circ}$ & $\%$ & $\mathrm{~N}^{\circ}$ & $\%$ \\
\hline 2-4 horas & 117 & 23,7 & 33 & 25,8 \\
\hline 5-10 horas & 45 & 9,1 & 17 & 13,3 \\
\hline 1 día & 231 & 46,8 & 51 & 39,8 \\
\hline 2 días & 101 & 20,4 & 27 & 21,1 \\
\hline TOTAL & 494 & $100 \%$ & 128 & $100 \%$ \\
\hline
\end{tabular}

Fuente: Investigación de campo.

Realizado por: Verónica Lema

El 47\% de los posibles clientes desean visitar Suscal por lo menos un día, el 24\% quieren permanecer de 2-4 horas, otro $20 \%$ por 2 días y el $9 \%$ restante de 5-10 horas. Los turistas extranjeros indican que el tiempo de visita en el cantón Suscal puede ser de un día con el 40\%, de 2-4 horas con el $26 \%$, dos días con el otro $21 \%$ y finalmente de 5-10 horas con un $13 \%$.

\section{Servicios que el turista desearía consumir en Suscal}

Tabla $\mathbf{N}^{\mathbf{0}}$ 12. Servicios preferidos por el turista nacional

\begin{tabular}{|c|c|c|c|c|}
\hline & \multicolumn{2}{|c|}{ Turista nacional } & \multicolumn{2}{c|}{ Turista internacional } \\
\hline Variables & $\mathrm{N}^{\circ}$ & $\%$ & $\mathrm{~N}^{\circ}$ & $\%$ \\
\hline Hospedaje & 134 & 27,1 & 44 & 34,4 \\
\hline Alimentación & 109 & 22,1 & 25 & 19,5 \\
\hline Guianza & 86 & 17,4 & 19 & 14,8 \\
\hline Transporte & 67 & 13,6 & 9 & 7,0 \\
\hline Información turística & 98 & 19,8 & 31 & 24,2 \\
\hline TOTAL & 494 & $100 \%$ & 128 & $100 \%$ \\
\hline
\end{tabular}

Fuente: Investigación de campo.

Realizado por: Verónica Lema.

Mediante la encuesta realizada a los turistas nacionales se determinó que el $27 \%$ prefieren el servicio de hospedaje, un 22\% alimentación, otro $20 \%$ desea que exista información turística, un 17\% quiere guianza y el 13\% transporte. El turista extranjero desea que el cantón Suscal cuente con el servicio de hospedaje en un 34\%, información turística con el $24 \%$, alimentación con un $20 \%$, guianza con otro $15 \%$ y finalmente transporte con el $7 \%$. 


\section{Gasto diario}

Tabla $\mathbf{N}^{\mathbf{0}}$ 13. Capacidad de gasto de los turistas nacionales

\begin{tabular}{|c|c|c|c|c|}
\hline & \multicolumn{2}{|c|}{ Turista nacional } & \multicolumn{2}{c|}{ Turista extranjero } \\
\hline Variables & $\mathrm{N}^{\circ}$ & $\%$ & $\mathrm{~N}^{\circ}$ & $\%$ \\
\hline$\$ 25-45$ & 285 & 57,7 & 51 & 39,8 \\
\hline$\$ 46-65$ & 118 & 23,9 & 46 & 35,9 \\
\hline \$ 66-85 & 73 & 14,8 & 22 & 17,2 \\
\hline Más de \$ 86 & 18 & 3,6 & 9 & 7,0 \\
\hline TOTAL & 494 & $100 \%$ & 128 & $100 \%$ \\
\hline
\end{tabular}

Fuente: Investigación de campo.

Realizado por: Verónica Lema.

Los posibles clientes indican que al momento de visitar Suscal prefieren en un 58\% gastar \$25-45, el 24\% de \$ 46-65, otro 15\% de \$ 66-85 y $3 \%$ restante de más de \$ 86 . Lo cual indica que el gasto promedio en cada viaje del turista es de 25-45 dólares. La capacidad de gasto del turista extranjero es en un 40\% de 25-45 dólares, el 36\% de 46-65 dólares, el 17\% de 66-85 dólares y el 7\% restante ms de 86 dólares.

\section{¿A través de qué medios de informa usted?}

Tabla Nº 14. Medios de información utilizada.

\begin{tabular}{|c|c|c|c|c|}
\hline \multicolumn{2}{|c|}{ Turista nacional } & \multicolumn{2}{c|}{ Turista extranjero } \\
\hline Variables & $\mathrm{N}^{\circ}$ & $\%$ & $\mathrm{~N}^{\circ}$ & $\%$ \\
\hline Guía turística & 66 & 13,4 & 34 & 26,6 \\
\hline Radio & 84 & 17,0 & 0 & 0,0 \\
\hline Televisión & 142 & 28,7 & 12 & 9,4 \\
\hline Internet & 139 & 28,1 & 71 & 55,5 \\
\hline Otros & 63 & 12,8 & 11 & 8,6 \\
\hline TOTAL & 494 & $100 \%$ & 128 & $100 \%$ \\
\hline
\end{tabular}

Fuente: Investigación de campo.

Realizado por: Verónica Lema.

El 29\% de los turistas encuestados mencionan que se informan a través de la televisión, seguido por el internet con un $28 \%$, el $17 \%$ con la radio, mientras que el $13 \%$ lo hace mediante una guía turística y otros medios como familiares, amigos y gente local. Todos los medios de información son relevantes al momento de promocionar un producto o servicio. Los medios de información utilizados por los turistas extranjeros es con el 55\% el internet, el 27\% usan guía turística y el $9 \%$ se informa a través de televisión y otros medios respectivamente, el uso de la radio es nulo para este segmento.

\section{g. Perfil del turista}

\section{1) Turista nacional}

El perfil del turista nacional está entre los 18 a 38 años de edad (45\%), el 52\% es de género masculino, la mayoría de los visitantes son de la 
región sierra 64\% y de la costa 34\%, el nivel de educación es secundaria $36 \%$.

El 42\% de los encuestados viajan generalmente por vacaciones y lo realizan en familia en un 47\%, un $43 \%$ viajan una vez al año,

El 22\% conoce este cantón y el 84\% está dispuesto en visitarlo, el 47\% están dispuestos permanecer un día, entre las actividades que desean realizar tenemos: degustar de la comida típica 20\%, caminata 18\%, turismo cultural 15\%, observación de flora y fauna 12\%, festividades locales 11\%, ciclismo 9\%, cabalgatas $8 \%$ y camping 7\%, además los servicios requeridos por el turista son: hospedaje 27\%, alimentación 22\%, información turística $20 \%$, guianza $17 \%$ y transporte $13 \%$.

El 58\% están dispuestos a pagar de 25 a 45 dólares por día y los medios de información que prefieren son la televisión 29\% y el internet 28\%.

\section{2) Turista internacional}

El perfil de los turistas internacionales está entre los 18 a 38 años de edad 46\%, el 51\% pertenece al género masculino la mayoría de los visitantes son de los países: EEUU 27\%, Alemania y Francia 19\%, el 73\% tienen educación de nivel superior.

El 98\% viajan generalmente por turismo, el $44 \%$ lo realiza entre amigos, el 44\% viajan dos veces al año.

El 95\% de los encuestados desconocen este cantón, al 67\% le gustaría visitarlo, las actividades de su preferencia son: observación de flora y fauna $32 \%$, turismo cultural 23\%, caminatas 16\%, comida típica 9\%, festividades locales y cabalgatas 6\% respectivamente, camping 5\% y ciclismo 3\%, los servicios requeridos son: hospedaje 34\%, información turística 24\%, alimentación 20\%, guianza 15\% y transporte $7 \%$.

El 40\% está dispuesto a pagar por día e 25 a 45 dólares, y el 55\% prefiere el internet.

\section{Proyección de la demanda y oferta}

\section{a) Proyección de la demanda potencial}

El 84\% de los turistas nacionales respondieron favorablemente con respecto a visitar este cantón, es decir:

$84182 * 84 \%=70713$ turistas potenciales nacionales

El $67 \%$ de los turistas extranjeros de igual manera se manifestaron positivamente en visitar el cantón, es decir:

$27147 * 67 \%=18188$ turistas potenciales extranjeros

Para la proyección de la demanda se utilizó el método de incremento compuesto, con el índice de incremento turístico del 7\% (Secretaria de Comunicación de la Presidencia, 2015), siendo este el rango el que evidencia 
realmente el crecimiento turístico, pues es un estimado de los últimos cinco años siendo la una cifra oficial. Se lo realizará para 5 años.

Tabla $N^{0} \mathbf{1 5}$. Proyección de la demanda potencial nacional y extranjera para 5 años.

\begin{tabular}{|c|c|c|c|}
\hline AÑO & $\begin{array}{c}\text { DEMANDA } \\
\text { NACIONAL }\end{array}$ & DEMANDA EXTRANJERA & TOTAL \\
\hline $2015(0)$ & 70713 & 18188 & 88901 \\
\hline $2016(1)$ & 75663 & 19461 & 95124 \\
\hline $2017(2)$ & 80959 & 20823 & 101782 \\
\hline $2018(3)$ & 86626 & 22281 & 108907 \\
\hline $2019(4)$ & 92690 & 23841 & 116531 \\
\hline $2020(5)$ & 99179 & 25510 & 124689 \\
\hline
\end{tabular}

Fuente: Investigación de campo.

Realizado por: Verónica Lema.

\section{b) Proyección de la oferta potencial}

Para la proyección de la oferta potencial se utilizó el método del incremento compuesto, se calculó tomando como base el número de clientes anuales del 2015 que utilizaron los diferentes servicios de alojamiento del cantón El Tambo y Cañar, dando un total de 23631 clientes, este valor se obtuvo del análisis de la oferta.

Tabla $\mathbf{N}^{\mathbf{0}}$ 16. Proyección de la oferta potencial.

\begin{tabular}{|c|c|}
\hline AÑO & DEMANDA NACIONAL \\
\hline $2015(0)$ & 23631 \\
\hline $2016(1)$ & 25285 \\
\hline $2017(2)$ & 27055 \\
\hline $2018(3)$ & 28949 \\
\hline $2019(4)$ & 30975 \\
\hline $2020(5)$ & 33144 \\
\hline
\end{tabular}

Fuente: Investigación de campo.

Realizado por: Verónica Lema.

Demanda potencial insatisfecha: Para calcular la demanda potencial insatisfecha se utilizó el total de los turistas potenciales, es decir la suma total de la demanda nacional y extranjera proyectada para los próximos 5 años restándole de la oferta potencial de cada año.

\section{Demanda insatisfecha proyectada}

Tabla $\mathbf{N}^{\circ}$ 17. Proyección de la demanda insatisfecha para el alojamiento.

\begin{tabular}{|c|c|c|c|}
\hline AÑO & $\begin{array}{c}\text { DEMANDA } \\
\text { POTENCIAL }\end{array}$ & $\begin{array}{c}\text { OFERTA } \\
\text { POTENCIAL }\end{array}$ & $\begin{array}{c}\text { DEMANDA } \\
\text { INSATISFECHA }\end{array}$ \\
\hline $2015(0)$ & 88901 & 23631 & 65270 \\
\hline $2016(1)$ & 95124 & 25285 & 69839 \\
\hline $2017(2)$ & 101782 & 27055 & 74727 \\
\hline $2018(3)$ & 108907 & 28949 & 79958 \\
\hline $2019(4)$ & 116531 & 30975 & 85556 \\
\hline $2020(5)$ & 124689 & 33144 & 91545 \\
\hline
\end{tabular}

Fuente: Investigación primaria.

Realizado por: Verónica Lema. 
Proyección demanda objetivo: Para calcular la demanda objetivo se consideró el 5\% de la demanda insatisfecha, teniendo en cuenta que este porcentaje permitirá dar inicio con el funcionamiento del proyecto turístico.

\section{Demanda objetivo proyectada}

Tabla $\mathbf{N}^{\mathbf{0}} \mathbf{1 8}$. Demanda objetivo proyectada

\begin{tabular}{|c|c|c|}
\hline AÑO & $\begin{array}{c}\text { DEMANDA } \\
\text { INSATISFECHA }\end{array}$ & $\begin{array}{c}\text { DEMANDA OBJETIVO } \\
(5 \%)\end{array}$ \\
\hline $2015(0)$ & 65270 & 3264 \\
\hline $2016(1)$ & 69839 & 3492 \\
\hline $2017(2)$ & 74727 & 3736 \\
\hline $2018(3)$ & 79958 & 3998 \\
\hline $2019(4)$ & 85556 & 4278 \\
\hline $2020(5)$ & 91545 & 4577 \\
\hline
\end{tabular}

Fuente: Investigación primaria.

Realizado por: Verónica Lema.

\section{Número de clientes proyectados para los próximos 5 años}

El número de clientes proyectados se pudo conocer a través de la demanda objetivo, la cual se calculó con el 5\% de la demanda insatisfecha, con la expectativa de iniciar sus actividades turísticas, de ahí se proyectó para los próximos 5 años, de esta manera se conoció el número de clientes anuales, mensuales semanales y diarios.

Tabla $\mathbf{N}^{0}$ 19. Número de clientes proyectados.

\begin{tabular}{|c|c|c|c|c|}
\hline AÑO & ANUAL & MENSUAL & SEMANAL & DIARIO \\
\hline $2015(0)$ & 3264 & 272 & 68 & 9 \\
\hline $2016(1)$ & 3492 & 291 & 73 & 10 \\
\hline $2017(2)$ & 3736 & 311 & 78 & 10 \\
\hline $2018(3)$ & 3998 & 333 & 83 & 11 \\
\hline $2019(4)$ & 4278 & 357 & 89 & 12 \\
\hline $2020(5)$ & 4577 & 381 & 95 & 13 \\
\hline
\end{tabular}

Fuente: Investigación primaria.

Realizado por: Verónica Lema.

\section{c) Proyección de las actividades de preferencia demandados por los turistas para los próximos 5 años}

Para conocer las actividades de preferencia por los turistas se tomó los datos obtenidos de las encuestas realizadas específicamente de la pregunta $\mathrm{N}^{\circ} 10$, los mismos que fueron proyectados de acuerdo al gusto de los turistas nacionales e internacionales. 


\section{1) Proyección de las actividades de preferencia demandados por los turistas nacionales}

Tabla $\mathbf{N}^{\mathbf{0}} \mathbf{2 0}$. Actividades demandadas por los turistas nacionales.

\begin{tabular}{|c|c|c|c|c|c|c|c|c|c|c|}
\hline \multicolumn{7}{|c|}{ SERVICIOS DE PREFERENCIA POR LOS TURISTAS NACIONALES } \\
\hline $\begin{array}{c}\text { AÑO } \\
\text { S }\end{array}$ & $\begin{array}{c}\text { Dema } \\
\text { nda } \\
\text { objeti } \\
\text { vo }\end{array}$ & $\begin{array}{c}\text { Dem } \\
\text { anda } \\
\text { objet } \\
\text { ivo } \\
\text { naci } \\
\text { onal } \\
80 \%\end{array}$ & $\begin{array}{c}\text { Cami } \\
\text { nata } \\
18 \%\end{array}$ & $\begin{array}{c}\text { Cabal } \\
\text { gata } \\
8 \%\end{array}$ & $\begin{array}{c}\text { Ciclis } \\
\text { mo } \\
9 \%\end{array}$ & $\begin{array}{c}\text { Camp } \\
\text { ing } \\
7 \%\end{array}$ & $\begin{array}{c}\text { Observa } \\
\text { ción de } \\
\text { flora y } \\
\text { fauna } \\
12 \%\end{array}$ & $\begin{array}{c}\text { Particip } \\
\text { ar en } \\
\text { festivid } \\
\text { ades } \\
\text { locales } \\
11 \%\end{array}$ & $\begin{array}{c}\text { Degu } \\
\text { star } \\
\text { de } \\
\text { comi } \\
\text { da } \\
\text { típica } \\
20 \%\end{array}$ & $\begin{array}{c}\text { Turis } \\
\text { mo } \\
\text { cultu } \\
\text { ral } \\
15 \%\end{array}$ \\
\hline $\begin{array}{c}2015 \\
(0)\end{array}$ & 3264 & 2611 & 470 & 209 & 235 & 183 & 313 & 287 & 522 & 392 \\
\hline $\begin{array}{c}2016 \\
(1)\end{array}$ & 3492 & 2794 & 503 & 223 & 251 & 196 & 335 & 307 & 559 & 419 \\
\hline $\begin{array}{c}2017 \\
(2)\end{array}$ & 3736 & 2989 & 538 & 239 & 269 & 209 & 359 & 329 & 598 & 448 \\
\hline $\begin{array}{c}2018 \\
(3)\end{array}$ & 3998 & 3198 & 576 & 256 & 288 & 224 & 384 & 352 & 640 & 480 \\
\hline $\begin{array}{c}2019 \\
(4)\end{array}$ & 4278 & 3422 & 616 & 274 & 308 & 240 & 411 & 376 & 684 & 513 \\
\hline $\begin{array}{c}2020 \\
(5)\end{array}$ & 4577 & 3662 & 659 & 293 & 330 & 256 & 439 & 403 & 732 & 549 \\
\hline
\end{tabular}

Fuente: Investigación primaria.

Realizado por: Verónica Lema

Este porcentaje se calculó teniendo como demanda potencial el valor de 88901 (100\%), de la cual la demanda potencial nacional es de 70713 (80\%) y la demanda potencial internacional es de 18188 (20\%).

Proyección de las actividades de preferencia demandados por los turistas internacionales

Tabla $\mathbf{N}^{\mathbf{2}}$ 21. Actividades de preferencia demandados por los turistas internacionales

\begin{tabular}{|c|c|c|c|c|c|c|c|c|c|c|}
\hline \multicolumn{8}{|c|}{ SERVICIOS DE PREFERENCIA POR LOS TURISTAS INTERNACIONALES } \\
\hline $\begin{array}{c}\text { AÑO } \\
\text { S }\end{array}$ & $\begin{array}{c}\text { Dema } \\
\text { nda } \\
\text { objeti } \\
\text { vo }\end{array}$ & $\begin{array}{c}\text { Dema } \\
\text { nda } \\
\text { objeti } \\
\text { vo } \\
\text { intern } \\
\text { acion } \\
\text { al } \\
20 \%\end{array}$ & $\begin{array}{c}\text { Cami } \\
\text { nata } \\
16 \%\end{array}$ & $\begin{array}{c}\text { Cabal } \\
\text { gata } \\
6 \%\end{array}$ & $\begin{array}{c}\text { Cicli } \\
\text { smo } \\
3 \%\end{array}$ & $\begin{array}{c}\text { Cam } \\
\text { ping } \\
5 \%\end{array}$ & $\begin{array}{c}\text { Observ } \\
\text { ación } \\
\text { de flora } \\
\text { y fauna } \\
32 \%\end{array}$ & $\begin{array}{c}\text { Partici } \\
\text { par en } \\
\text { festivid } \\
\text { ades } \\
\text { locales } \\
6 \%\end{array}$ & $\begin{array}{c}\text { Degu } \\
\text { star } \\
\text { de } \\
\text { comi } \\
\text { da } \\
\text { típica } \\
9 \%\end{array}$ & $\begin{array}{c}\text { Turis } \\
\text { mo } \\
\text { cultu } \\
\text { ral } \\
23 \%\end{array}$ \\
\hline $\begin{array}{c}2015 \\
(0)\end{array}$ & 3264 & 653 & 104 & 39 & 20 & 33 & 209 & 39 & 59 & 150 \\
\hline $\begin{array}{c}2016 \\
(1)\end{array}$ & 3492 & 698 & 112 & 42 & 21 & 35 & 223 & 42 & 63 & 161 \\
\hline $\begin{array}{c}2017 \\
(2)\end{array}$ & 3736 & 747 & 120 & 45 & 22 & 37 & 239 & 45 & 67 & 172 \\
\hline
\end{tabular}




\begin{tabular}{|c|c|c|c|c|c|c|c|c|c|c|}
\hline $\begin{array}{c}2018 \\
(3)\end{array}$ & 3998 & 800 & 128 & 48 & 24 & 40 & 256 & 48 & 72 & 184 \\
\hline $\begin{array}{c}2019 \\
(4)\end{array}$ & 4278 & 856 & 137 & 51 & 26 & 43 & 274 & 51 & 77 & 197 \\
\hline $\begin{array}{c}2020 \\
(5)\end{array}$ & 4577 & 915 & 146 & 55 & 27 & 46 & 293 & 55 & 82 & 211 \\
\hline
\end{tabular}

Fuente: Investigación primaria.

Realizado por: Verónica Lema.

Este porcentaje se calculó teniendo como demanda potencial el valor de 88901 (100\%), de la cual la demanda potencial nacional es de 70713 (80\%) y la demanda potencial internacional es de 18188 (20\%).

\section{Proyección de los servicios de preferencia demandados por los turistas para los próximos 5 años}

Para conocer los servicios de preferencia de los turistas se tomó los datos específicamente de la pregunta $\mathrm{N}^{\circ} 12$, y se los proyectó.

\section{CONCLUSION profundice la determinación comercial}

Mediante la validación del potencial turístico del cantón Suscal se pudo evidenciar que posee una gran riqueza en cuanto al patrimonio cultural inmaterial ya que conserva una significativa memoria ancestral las cuales se han mantenido vigentes hasta hoy, también se pudo encontrar atractivos naturales de jerarquía I con un 14\% y un 86\% de jerarquía II; lo cual indica que la zona posee potencialidad y que son factores clave para que el área de estudio sea un producto turístico clave para la visita de turistas nacionales y extranjeros, además para la captación de potenciales clientes.

El producto turístico es comercialmente viable, debido a que existe una aceptación alta por parte de los turistas nacionales en un 84\% y de extranjeros en un 67\%, lo cual constituye una gran oportunidad para emprender negocios turísticos con la posibilidad de promocionar y a futuro posicionar al cantón como destino de visita.

Los productos turísticos solicitados por los encuestados nacionales han sido: degustar de la comida típica 20\%, caminata 18\%, turismo cultural $15 \%$, observación de flora y fauna $12 \%$, festividades locales $11 \%$, ciclismo $9 \%$, cabalgatas $8 \%$ y camping $7 \%$, además los servicios requeridos por el turista son: hospedaje 27\%, alimentación 22\%, información turística 20\%, guianza $17 \%$ y transporte $13 \%$.

Los productos turísticos solicitados por los encuestados extranjeros (EEUU 27\%, Alemania y Francia 19\%) fueron: observación de flora y fauna $32 \%$, turismo cultural 23\%, caminatas 16\%, comida típica 9\%, festividades locales y cabalgatas 6\% respectivamente, camping 5\% y ciclismo 3\%, los 
servicios requeridos son: hospedaje 34\%, información turística 24\%, alimentación $20 \%$, guianza $15 \%$ y transporte $7 \%$.

Los productos solicitados avizoran un margen de éxito representativo basados en los valores de disposición a pagar: extranjeros el $40 \%$ está dispuesto a pagar por día 25 a 45 dólares, y los nacionales el 58\% están dispuestos a pagar de 25 a 45 dólares por día. Sin embargo se recomienda realizar un estudio de factibilidad técnica integral para determinar la viabilidad del proyecto.

De lo que se deduce que los productos ofertados deben tener un rango amplio de audiencia, si bien es cierto que mayoritariamente los turistas que llegan al destino son jóvenes (nacional entre los 18 a 38 años de edad (45\%) y extranjero 18 a 38 años de edad 46\%), los paquetes deberán encaminarse a actividades que pueden ser practicadas por este segmento, también los servicios requeridos como alimentación, hospedaje y transporte dejan la puerta abierta a otros segmentos de mayor edad que puedan disfrutarlos adicionales a la cultura del lugar.

La comercialización del producto se realizara basados en el Marketing Mix del producto considerando: Consumidor, Costo, Conveniencia, Comunicación. Lo que incide directamente en la decisión final del cliente. Este apartado se detalla a profundidad en la segunda fase del proyecto.

\section{References:}

Armijo M, (2009). Manual de planificación estratégica e indicadores de desempeño en el sector público. ILPES/CEPAL. Consultado el 24 de diciembre del 2015.2 Recuperado de:http://www.cepal.org/ilpes/noticias/paginas/3/38453/manual_planificacio n_estrategica.pdf

Asamblea Nacional del Ecuador. (2008). La Constitución de la República del Ecuador. Derechos de las comunidades, pueblos y nacionalidades Capítulo cuarto. Artículo 57. Literal 12.

Barrado D. (2002). La articulación territorial del turismo: apuntes desde la perspectiva territorial y la sectorial, Cuadernos de Ordenación del Territorio, No 3. Cuarta edición. pp. 3-7.

Calderón Á. (2015). Análisis de la cadena del turismo. Comisión Económica para América Latina y el Caribe-CEPAL. Quito-Ecuador

Dores, M. (2011). Identificación y documentación del patrimonio cultural inmaterial y análisis de riesgo del PCI. Cusco: CRESPIAL. p.4.

García N. (1999). Los usos sociales del Patrimonio Cultural. Patrimonio Etnológico. Departamento de Antropología de la UAM-Iztapalapa. México 
Gobierno Autónomo Descentralizado Intercultural Participativo Comunitario Suscal-GADIPCS. (2013). Plan de ordenamiento y desarrollo territorial .Informe final. Suscal.

Hernández R. (2007). Modelos de cadena de valor y desarrollo de destinos turísticos. Una aplicación a Maspalomas. Facultad de Ciencias Económicas y Empresariales. Universidad de La Laguna. España.

Instituto Nacional de Patrimonio Cultural-INPC. (2011). Instructivo para fichas de registro e inventario del Patrimonio Cultural Inmaterial. QuitoEcuador. Ediecuatorial.

Instituto Nacional del Patrimonio Cultural-INPC. (2011). Instructivo de registro e inventario de patrimonio cultural. Informe final. Quito.

Mideros A. (2015). Economía solidaria: crecer redistribuyendo la riqueza. Revista CHAKANA. Ed 8: 24/08/2015. SENPLADES. Consultado el 6 de julio del 2016. Recuperado de: http://www.planificacion.gob.ec/wpcontent/uploads/downloads/2015/11/CHAKANA8.pdf

Ministerio de Coordinación de la Producción, Empleo y CompetitividadMCPEC. (2011). Agenda territorial de la provincia del Cañar. Consultado el 30 de Agosto de 2015. Recuperado de: http://www.produccion.gob.ec/wpcontent/uploads/downloads/2013/02/AGENDA-TERRITORIAL-

CA\%C3\%91AR.pdf

Ministerio de Turismo-MINTUR. (2004). Metodología para inventarios de atractivos turísticos. Quito. Consultado el 30 de Agosto de 2015. Recuperado de http://www.mintur.gov.ec

Ministerio de Turismo-MINTUR. (2014). Turismo es el cuarto rubro que aporta a la economía: Quito. Consultado el 27 de Agosto de 2015. Recuperado de: http://www.turismo.gob.ec/resumen-2014-turismoecuatoriano-en-su-mejor-momento/

Mullo, J. (2007). Danza y música en la fiesta del Ecuador. Ritualidad, simbología y diversidad en la construcción de la identidad indígena, negra y montubia. Revista 192 Nacional de Cultura Encuentros $\mathrm{N}^{\circ} 11$. Consejo Nacional de Cultura. Quito - Ecuador.

Organización Mundial de Turismo-OMT. (2014). ¿Por qué el turismo?. Consultado el 8 de Agosto de 2015. Recuperado de: www2.unwto.org/es/content/por-que-el-turismo

Organización Mundial del Turismo-OMT. (2015). Panorama OMT del turismo internacional, edición 2015. Consultado el 6 de julio del 2016. Recuperado de: http://www.e-unwto.org/doi/pdf/10.18111/9789284416875

Plan de Desarrollo y Ordenamiento Territorial de la provincia del Cañar 2015-2019-PDyOT. (2015). Componente socio cultural. Consultado el 06 de julio del 2016. Recuperado de: http://app.sni.gob.ec/snilink/sni/PORTAL_SNI/data_sigad_plus/sigadplusdocumentofinal/03600001 
50001_Plan\%20de\%20desarrollo\%20y\%20ordenamiento\%20territorial_0408-2015_10-08-14.pdf

Secretaria Nacional de Planificación y Desarrollo- SENPLADES. (2012). Transformación de la matriz productiva, revolución productiva a través del conocimiento y el talento humano. Primera edición. SENPLADES. QuitoEcuador. pp. 11, 12.

Secretaria Nacional de Planificación y Desarrollo. (2015). Plan Estratégico SENPLADES 2014-2017. Consultado el 19 de octubre del 2015. Recuperado de: http://www.planificacion.gob.ec/wpcontent/uploads/downloads/2015/10/Plan-Estrategico-Senplades-20142017.pdf

Torres V. (2008). Políticas públicas, territorios y localidades. Ecuador: innovación municipal e integración municipal. Primera edición. Abya-Yala. Quito-Ecuador. pp. 39, 40. 